\title{
Time Series Phase Unwrapping Based on Graph Theory and Compressed Sensing
}

\author{
Zhang-Feng $\mathrm{Ma}^{\circledR}$, Student Member, IEEE, Mi Jiang ${ }^{\circledR}$, Senior Member, IEEE, \\ Mostafa Khoshmanesh, and Xiao Cheng
}

\begin{abstract}
Time Series SAR interferometry (InSAR) (TS-InSAR) has been widely applied to monitor the crustal deformation with centimeter- to millimeter-level accuracy. Phase unwrapping (PU) errors have proven to be one of the main sources of bias that hinder achieving such high accuracy. In this article, a new time series PU approach is developed to improve the unwrapping accuracy. The rationale behind the proposed method is to first improve the sparse unwrapping by mitigating the phase gradients in a 2-D network and then correcting the unwrapping errors in time, based on the triplet phase closure. Rather than the commonly used Delaunay network, we employ the all-pairs-shortest-path (APSP) algorithm from graph theory to maximize the temporal coherence of all edges and to approach the phase continuity assumption in the 2-D spatial domain. Next, we formulate the PU error correction in the 1-D temporal domain as compressed sensing (CS) problem, according to the sparsity of the remaining phase ambiguity cycles. We finally estimate phase ambiguity cycles by means of integer linear programming (ILP). The comprehensive comparisons using synthetic and real Sentinel-1 data covering Lost Hills, California, confirm the validity of the proposed 2-D + 1-D unwrapping approach and its superior performance compared to previous methods.
\end{abstract}

Index Terms-All-pairs-shortest-path (APSP), compressed sensing (CS), graph theory, phase unwrapping (PU), SAR interferometry (InSAR), time series.

\section{INTRODUCTION}

$\mathbf{P}$ HASE unwrapping (PU) is a key step in SAR interferometry (InSAR) time series processing for high-precision deformation monitoring. The aim of PU is to recover the proper ambiguity number of the $2 \pi$ phase cycles from the

Manuscript received December 7, 2020; revised January 27, 2021 and March 1, 2021; accepted March 13, 2021. This work was supported in part by the National Natural Science Foundation of China under Grant 41774003 and Grant 42074008, in part by the European Space Agency (ESA), Ministry of Science and Technology (MOST) of China Dragon 5 Project under Grant 59332, and in part by the Program of China Scholarship Council under Grant 202006710013. (Corresponding author: Mi Jiang.)

Zhang-Feng $\mathrm{Ma}$ is with the School of Earth Sciences and Engineering, Hohai University, Nanjing 211100, China (e-mail: jspcmazhangfeng@ hhu.edu.cn).

Mi Jiang and Xiao Cheng are with the School of Geospatial Engineering and Science, Sun Yat-sen University, Guangzhou 510275, China, and also with the Southern Marine Science and Engineering Guangdong Laboratory, Zhuhai 519082, China (e-mail: jiangmi@mail.sysu.edu.cn; chengxiao9@ mail.sysu.edu.cn).

Mostafa Khoshmanesh is with the Department of Mechanical and Civil Engineering, California Institute of Technology, Pasadena, CA 91125 USA (e-mail: mkh@ caltech.edu).

Color versions of one or more figures in this article are available at https://doi.org/10.1109/TGRS.2021.3066784.

Digital Object Identifier 10.1109/TGRS.2021.3066784 interferogram, in which the observations are known modulo$2 \pi[1]-[3]$. The main challenge of PU is that it is extremely difficult to, first, distinguish which pixel contains phase cycles, and second, how many the cycles are. A certain phase continuity assumption that the local phase gradient between neighboring points should be smooth and less than $\pi$ makes this problem tractable.

Based on this assumption, $L^{\mathrm{p}}$-Norm [4] methods such as least squares, minimum cost flow (MCF) [5], and minimum discontinuity [6] attempt to unwrap phases by shrinking the Euclidean distances or minimizing the difference between the gradient of the wrapped phase and the unknown true value. Despite evident success in various applications, this hypothesis might not be valid in real SAR interferograms due to the phase discontinuities caused by phase noise and fast phase variation of the signal of interest due to deformation or local topography.

In order to identify the phase discontinuities, different strategies have been proposed, generally resorting to the temporal information. In 3-D methods, the phase continuity assumption is extended to three dimensions, the third being time. For example, the 1-D + 2-D method presented by Pepe and Lanari [7] integrates the temporal MCF network programming with the spatial PU to mitigate phase gradients and therefore to approach the phase continuity assumption. Costantini et al. [8] proposed a multi-dimensional PU procedure, in which redundant phase gradients both in temporal and spatial dimensions are utilized in optimizing the 3-D irrotationality constraints. Furthermore, Hooper and Zebker [9] proposed a QUASI- $L^{\infty}$-Norm 3-D PU algorithm, which extends the 2-D branch-cut theory to 3-D through linking phase residuals to construct a $3-\mathrm{D}$ discontinuity surface. Although 3-D-based approaches are more likely to achieve reliable results, heavy computational burden and the error propagation induced by misestimating the phase residues or gradients may degrade the unwrapping efficiency.

Recent studies have demonstrated that the phase closure test for PU error detection and correction is a powerful method [10]. The rationale behind this method is that a redundant temporal network of interferograms allows evaluating the errors by checking the consistency of triplets of spatially unwrapped phases. After establishing a linear relationship between required integer ambiguities and unwrapped phase observations (see Section III-A), a least-squares adjustment is implemented on a pixel-by-pixel basis in space. Note that typically all of the least-squares estimates will be nonzero. This 
interprets the final model challenging as the solution should be sparse, i.e., only a fraction of the unwrapped interferogram series should include unwrapping errors [11]. To solve this issue, $\mathrm{Xu}$ and Sandwell [12] modified the objective function by taking advantage of the least absolute shrinkage and selection operator (Lasso) [13], which is a $L^{1}$-norm regularization. Indeed, Lasso is equivalent to a Laplacian-like prior [13] and yields sparse solution vectors, having only some coordinates that are nonzero. However, the Lasso regularization is not only very time-consuming for the penalty selection and the iterative solver, but also needs parameterization. Furthermore, the solutions in least-squares and Lasso need to be rounded to their nearest integer, which is obviously undesirable in strong noise scenarios. In this context, the discontinuous $L^{0}$-Norm is more effective for recovering sparse signals as the solution can provide the minimum number of corrections required for global loop closure.

The main objective of this article is to improve the PU error correction algorithm. Given that $L^{0}$-Norm is not convex, making the minimization very computationally challenging, we reformulate the problem of integer ambiguity correction under the mathematical framework of compressed sensing (CS) technique and replace the nonconvex discontinuous $L^{0}$-norm by the convex continuous $L^{1}$-norm, leading to a $L^{1}$-norm integer linear programming (ILP). Because the spatial unwrapping errors will affect the temporal phase closure, and consequently the sparsity of integer ambiguities to be estimated, we also investigate the 2-D PU on the sparse grid, with emphasis on the spatial reference network. As a result, time-series PU becomes the focus of this article.

The rest of this article is organized as follows. Section II introduces the spatial 2-D PU based on the all-pairs-shortestpath (APSP) algorithm. Section III is dedicated to the unwrapping error correction using the CS technique. We then test the performance of the developed algorithm and compare the results with those from the state-of-the-art techniques, using synthetic and real data in Sections IV and V, respectively. Finally, conclusions are outlined in Section VI.

\section{SPARSE PU (SPU) BASED ON APSP AlgORITHM}

SPU aims to recover phase ambiguities from all edges of a network consisting of sparsely coherent points. The successful unwrapping relies on a priori phase continuity assumption that the phase difference on an arbitrary edge is less than $\pi$. The Delaunay triangulation is a commonly used method to connect all sparse points. However, the algorithm seeks whether the convex hull of two neighboring triangles contains other points and whether triangles overlap and, hence, is independent of the rule of phase continuity. This implies many edges may have higher phase differences in a spatial network when deformation signals, atmospheric turbulence, and phase noise are present in the interferogram.

When time series are available, the temporal coherence is a valid and effective measure to quantify the phase difference [14], [15]. The temporal coherence is defined as

$$
\rho=\frac{1}{M}\left|\sum_{i=1}^{M} e^{j \Delta \phi_{i, p, q}}\right|
$$

where $\Delta \phi_{i, p, q}$ is the phase difference between vertex $p$ and $q$ in an interferogram $i$. The higher values of temporal coherence represent that an edge has a smaller phase variation in the temporal dimension. Accordingly, it is favorable to select edges with higher temporal coherence to improve the unwrapping results for all the interferograms, instead of using the edges simply obtained from the Delaunay triangulation algorithm. To this end, the APSP algorithm can be employed [16], which has been used in [17] for the deformation rate estimation.

Given a weighted and undirected graph $G=\{V, E\}$ with weight function $w: E \rightarrow R^{+}$, where $V$ is the set of all vertices (i.e., sparse points $v_{1}, v_{2}, \ldots, v_{\mu}$ ) and $E$ represents the set of edges formed under a certain constraint, the APSP algorithm finds edges connecting source $v_{i}$ and terminal $v_{j}$ in the set $E$ with the minimum weight. This procedure is recursive and the path connecting pair $\left(v_{i}, v_{j}\right)$ is refined step by step, while only saving the edges with the best temporal coherence found in each iteration.

An initial list of pairs $\left(v_{i}, v_{j}\right)$ needs to be prepared first. For this purpose, we use the Delaunay triangulation and k-nearest neighbors (KNN, $\mathrm{K}=100)$ algorithms to create two sets of sources and terminals. The initial set of edges $(E)$ is then created by combining all the edges in the KNN network and Delaunay triangulation (see Fig. 1). After estimating the weight $w$ for each edge in the pair list of set $E$ as $w=$ $-10 \log 10(\rho)$, where $\rho$ is the temporal coherence defined in (1), the edge $\left(v_{i}, v_{j}\right)$ is replaced by the lower-weight edges in $E$. For instance, the edge between vertex $\left(v_{1}, v_{2}\right)$ in the pair list with weight $w_{12}$ can be substituted with edges $\left(v_{1}, v_{3}\right)$ and $\left(v_{3}, v_{2}\right)$, with respective weights of $w_{13}$ and $w_{32}$, where $w_{12}>w_{13}+w_{32}$.

Once all edges are updated using the APSP algorithm, the total temporal coherence of the newly generated network is maximized (see Fig. 1). Given such an APSP network, the MCF algorithm can be implemented to obtain the phase ambiguities. Nevertheless, here we use a slightly different form of the MCF, based on the triangle irrotationality constraint for Delaunay triangulations.

For a Delaunay network of $M$ triangles and $N$ edges, the MCF objective function is formulated as

$$
\begin{aligned}
& {\left[A_{M \times N}-A_{M \times N}\right]\left[\begin{array}{l}
K_{N \times 1}^{+} \\
K_{N \times 1}^{-}
\end{array}\right]=U_{M \times 1}} \\
& \quad \arg \min \left\{f_{1 \times N}^{+} K_{N \times 1}^{+}+f_{1 \times N}^{-} K_{N \times 1}^{-}\right\} K^{+}, K^{-} \in \mathbb{N}^{0} .
\end{aligned}
$$

In which, $K^{+}$and $K^{-}$are two slack vectors for edge ambiguities to be computed, $f^{+}$and $f^{-}$are probability cost for those two slack vectors, and $A$ is the design matrix, which is determined by all triangles in Delaunay, defined as

$$
\begin{aligned}
A_{M \times N}= & {\left[\begin{array}{ccccc}
a_{11} & a_{12} & a_{13} & \cdots & a_{1 N} \\
a_{21} & a_{22} & a_{23} & \cdots & a_{2 N} \\
\vdots & \vdots & \vdots & \vdots & \vdots \\
a_{M 1} & a_{M 2} & a_{M 3} & \cdots & a_{M N}
\end{array}\right] } \\
\text { s.t. } a_{i j}= & \left\{\begin{array}{ccc}
-1, & \text { if } E_{j} \text { is counter-clockwise direction in } \operatorname{Tr}_{i} \\
1, & \text { if } E_{j} \text { is clockwise direction in } \operatorname{Tr} i_{i} \\
0, & \text { otherwise }
\end{array}\right. \\
& (i=1,2,3, \ldots, M ; j=1,2,3, \ldots, N) .
\end{aligned}
$$




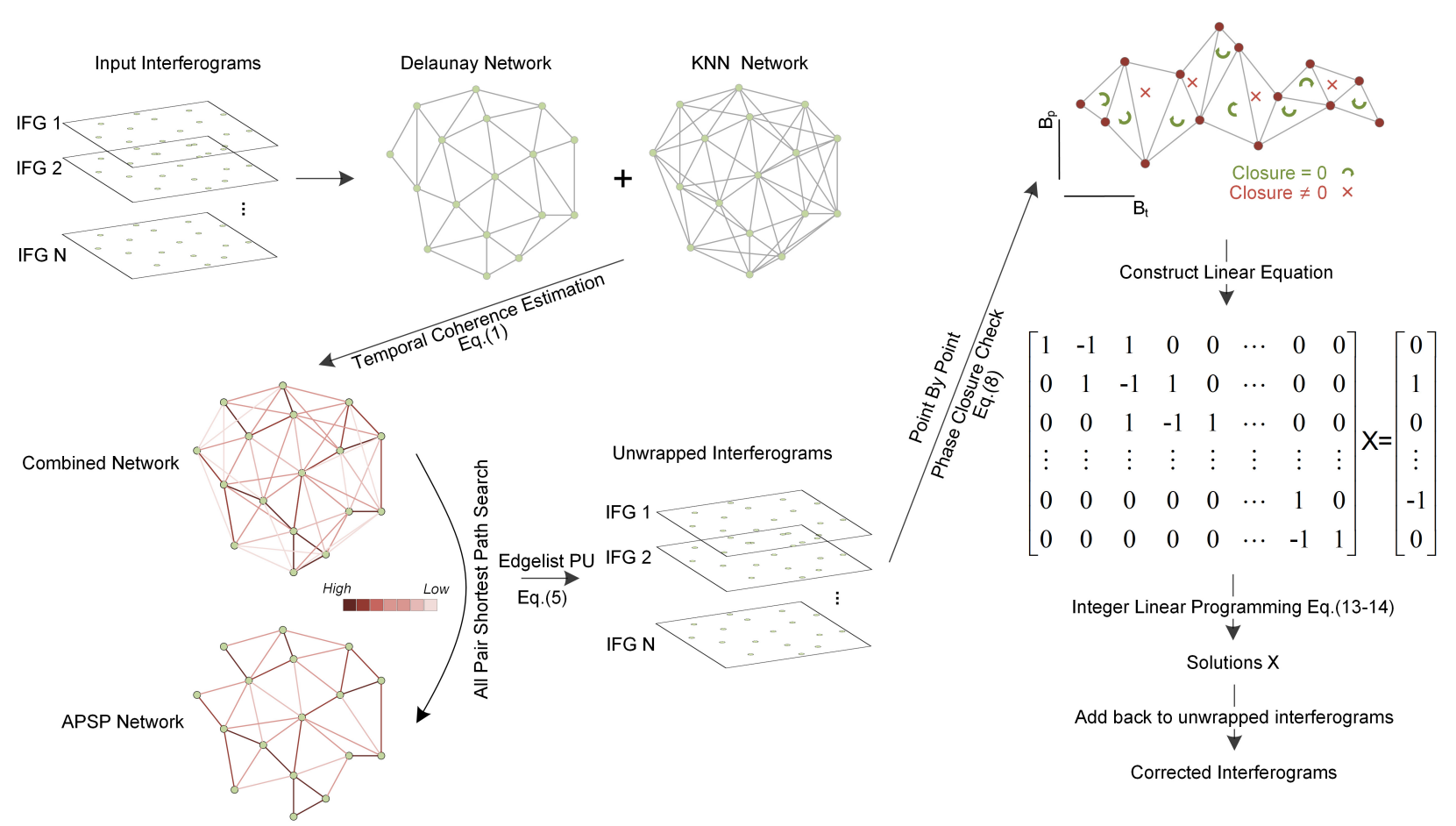

Fig. 1. Workflow diagram of sparse phase unwrapping based on APSP network and phase unwrapping error correction based on ILP. lows:

The triangle irrotationality constraint $U$ is defined as fol-

$$
\begin{aligned}
U_{i} & =\sum_{j=1}^{N} a_{i j} \cdot n_{j} \\
\text { s.t. } n_{j} & =\operatorname{round}\left(-\delta \phi_{j} / 2 \pi\right)
\end{aligned}
$$

where $\delta \phi_{j}$ is the phase difference of $E_{j}$. When an optimal solution of $\mathrm{K}^{+}$and $\mathrm{K}^{-}$is found, ambiguities for all edges are calculated as $K^{+}-K^{-}$. Next, the ambiguities estimated for connected edges are integrated through the flood-fill procedure to determine the ambiguity cycles of all points.

The APSP network, however, is different from one formed by Delaunay triangulation, in that some of the triangles are eliminated, making the triangle irrotationality constraint infeasible. We solve this issue by converting the constraint from the triangles to the edges, using the edgelist PU algorithm presented in [18]. More specifically, for an APSP network with $N$ edges and $P$ points, the MCF objective function is defined as

$$
\begin{gathered}
{\left[\begin{array}{lll}
A_{N \times P}-A_{N \times P} & I_{N \times N}-I_{N \times N}
\end{array}\right]\left[\begin{array}{l}
m_{P \times 1}^{+} \\
m_{P \times 1}^{-} \\
K_{N \times 1}^{+} \\
K_{N \times 1}^{-}
\end{array}\right]=S_{N \times 1}} \\
\arg \min \left\{f_{1 \times N}^{+} K_{N \times 1}^{+}+f_{1 \times N}^{-} K_{N \times 1}^{-}\right\} \\
m^{+}, m^{-}, K^{+}, K^{-} \in \mathrm{N}^{0}
\end{gathered}
$$

where $I$ is the unit matrix, $m^{+}$and $m^{-}$are two slack vectors for point ambiguities, and $A$ is the design matrix defined as

$$
A_{N \times P}=\left[\begin{array}{ccccc}
a_{11} & a_{12} & a_{13} & \cdots & a_{1 P} \\
a_{21} & a_{22} & a_{23} & \cdots & a_{2 P} \\
\vdots & \vdots & \vdots & \vdots & \vdots \\
a_{N 1} & a_{N 2} & a_{N 3} & \cdots & a_{N P}
\end{array}\right]
$$

$$
\begin{aligned}
\text { s.t. } a_{i j}= & \begin{cases}1, & \text { if } \mathrm{j} \text { is starting node in } E_{i} \\
-1, & \text { if } \mathrm{j} \text { is destination node in } E_{i} \\
0, & \text { otherwise }\end{cases} \\
& (i=1,2,3, \ldots, N ; j=1,2,3, \ldots, P) .
\end{aligned}
$$

Moreover, the edge constraint $S$ of $E_{i}$ is defined as $S_{i}=$ $\operatorname{round}\left(-\delta \phi_{i} / 2 \pi\right)$ where $\delta \phi_{i}$ is the phase difference of $E_{i}$. When the MCF method obtains the optimal solution using (5), point ambiguities are estimated as $m^{+}-m^{-}$and, hence, the flood-fill operation is not required. It, therefore, reduces the computational burden for the PU process.

In summary, APSP-Based SPU is composed of four steps as shown in the workflow diagram (see Fig. 1): 1) generation of the Delaunay triangulation and KNN networks; 2) calculating temporal coherence for the combined network using (1); 3 ) forming the APSP network based on the temporal coherence; and 4) retrieval of the phase ambiguities on all the sparse points using the edgelist PU algorithm: (5).

\section{PU ERror Correction USING CS}

\section{A. Phase Ambiguity Correction}

Recent studies have shown the effectiveness of triplet phase closure in unwrapping error detection [10], [11]. Given a triangle loop of three unwrapped phases $\psi_{m, j}, \psi_{j, k}$ and $\psi_{m, k}$ obtained from the interference of three acquisitions $m, j$, and $k$, the phase closure $\Delta \psi_{m, j, k}$ is defined as

$$
\Delta \psi_{m, j, k}=\psi_{m, j}+\psi_{j, k}-\psi_{m, k} .
$$

The non-zero $\Delta \psi_{m, j, k}$ represents errors, which may be caused by the decorrelation noise, multi-looking, filtering, and/or PU errors. Assuming PU errors as the sole cause of non-zero $\Delta \psi_{m, j, k}$, the phase closure can be expressed 
as [11], [14]

$$
\Delta \psi_{m, j, k}=\psi_{m, j}+\psi_{j, k}-\psi_{m, k}-\operatorname{wrap}\left(\psi_{m, j}+\psi_{j, k}-\psi_{m, k}\right)
$$

where the wrap is the wrapping operator. $\Delta \psi_{m, j, k}=0$ denotes no PU error for all the three unwrapped interferograms, while $\Delta \psi_{m, j, k} \neq 0$ shows PU errors in at least one interferogram. The residual phase ambiguity in the triangle loop is expressed as $U_{m, j, k}=\operatorname{round}\left(-\Delta \psi_{m, j, k} / 2 \pi\right)$.

Based on the concept of phase closure, an $L^{2}$-norm method is proposed to handle the PU errors. In this method, the correction process is defined as

$$
\arg \min \left\{\|G X-U\|_{2}\right\} G X=U \Rightarrow\left[\begin{array}{l}
A \\
D
\end{array}\right] X=U
$$

where $G$ is the design matrix of this optimization problem, and $A$ is the incidence matrix representing the triangle loops. $X$ and $U$ are the ambiguity vector and phase closure, respectively. Note that $A$ is a $T \times N$ matrix, where $T$ represents the number of triangle loops and $N$ denotes the number of interferograms. In the SBAS network, $T$ is always smaller than $N$, meaning that the number of loops is less than the number of interferograms. Thus, $A$ is a rank-deficient matrix. Given a certain assumption that some of the pixels contain no PU errors, the phase ambiguity of those pixels should be set to zero. Therefore, $D$ is added to constrain part of the phase ambiguity to be zero. Details of the construction of $A$ and $D$ matrices are described in Appendix A.

The introduction of $D$ can increase the rank of $G$. However, the combination of $A$ and $D$ still cannot make the matrix $G$ full-rank when unwrapping errors occur in quite a few interferograms. To overcome this issue, a Lasso [12] method, integrating $L^{2}$-norm with $L^{1}$-norm regularization was proposed. Its objective function is defined as

$$
\arg \min \left\{\|A X-U\|_{2}+\alpha\|X\|_{1}\right\}
$$

in which, the Lagrange multiplier $\alpha$ is used to ensure that optimization of (10) is feasible by bounding $\|X\|_{1}$ to less than a fixed value, by forcing part of $X$ to be zero. It also eliminates the necessity to set up the constraint matrix $D$. However, the parameterization and selection of $\alpha$ is time-consuming.

Moreover, limited to the global smoothness of L2-form, the above-mentioned methods both need a risky round operator to obtain the integer solutions. To illustrate, 0.5 would be rounded to 1 .

\section{B. CS and ILP}

As stated above, the phase ambiguity correction is an ill-conditioned problem for $T \ll N$, and integer solutions are expected to be directly obtained. In order to cope with this issue, we propose to solve this problem based on the CS framework. Compared to the conventional recovery techniques [19], CS [20]-[22] seeks to recover signals from fewer samples than those required by the Nyquist rate. This issue can be effectively resolved only if: 1) solutions are sparse and $k<\operatorname{Spark}(A) / 2$, where $k$ is the number of non-zero elements in the solution vector and Spark denotes the smallest rank of columns and rows and 2) the incidence matrix $A$ fulfills the restricted isometry property (RIP) condition [23], meaning the incidence matrix and its transform base should be incoherent. It is still an elusive topic in information theory to determine which ensembles can satisfy the RIP condition with high probability.

In the case of a redundant SBAS graph, however, it is possible to check whether the incidence matrix meets the aforementioned criteria [24]. The spark of $A$ is $T$ (the number of triangle loop in SBAS graph) [25], [26], and therefore the sparsity must follow $k<T / 2$. Otherwise, the signal recovery degree will be greatly affected. As mentioned in Section III-A, some pixels in the interferograms contain no PU errors, and the phase ambiguity of those pixels is zero. In this case, CS can be used to recover the phase ambiguity. For every $k$-sparse solution vector, $X$ is the unique solution to the $L^{0}$ optimization criterion

$$
\arg \min \|X\|_{0} \text { s.t. } A X=U \text {. }
$$

However, (11) cannot be solved in a polynomial time [27]. To overcome this challenge, a common strategy that converts (11) to a convex optimization based on $L^{1}$-Norm is given by [28], [29]

$$
\arg \min \left\|f^{T} X\right\|_{1} \text { s.t. } A X=U
$$

where $f^{T}$ is the reciprocal of the temporal coherence vector, and (12) can be treated as a linear programming (LP) problem [29]. Given the integer property of solutions, an ILP method based on LP is proposed to solve (12), which can directly obtain the integer solution, while (9) and (10) can only obtain the non-integer solutions and cannot avoid the risky rounding operation.

In this contribution, we follow the basic concepts of $L^{1}$-Norm and its implementation from Marshall and Bethel [30]. The overall features of our proposed approach are compared with the other methods in Table I. Notably, obtaining an integer solution for the phase ambiguity correction problem, to the best of our knowledge, is proposed and discussed for the first time in this study.

Before searching for an ILP solution, we need to reformulate the objective function of $L^{1}$-norm for phase ambiguity correction, (12), in a way that all the parameters to be estimated are non-negative. In order to do this, we introduce two slack vectors $X^{+}$and $X^{-}$for phase ambiguity $X$. Rewriting (12) in terms of these slack vectors into

$$
[A-A]\left[\begin{array}{l}
X^{+} \\
X^{-}
\end{array}\right]=U X^{+}, X^{-} \in \mathrm{N}^{0} ; X^{+}, X^{-} \leq l_{u p}
$$

toward the minimization of

$$
\arg \min \left\|f^{T}\left(X^{+}-X^{-}\right)\right\|_{1}
$$

where $l_{\text {up }}$ is the upper boundary of the solutions.

It is a difficult task to solve (13) and (14) given the extreme computational complexity of ILP. Instead, three different methods have been explored to find a reliable solution [31]: 1) enumeration techniques such as the well-known branchand-bound (BB) algorithm; 2) cutting plane techniques; and 3 ) heuristic techniques. These algorithms generally seek to find 
TABLE I

COMPARISON OF UnWRAPPING ERROR CORRECTION METHOdS

\begin{tabular}{ccccccc}
\hline Method & Error Criterion & Solutions & Computation & Exempt of Full Rank & Solution Type & Estimation Efficiency \\
\hline Fattahi & $\arg \min \|G X-U\|_{2}$ & LS & Non-Iterative & No & Non-Integer & Yes \\
$\begin{array}{c}\text { Xu et al. \& Zhang et } \\
\text { al. }\end{array}$ & $\arg \min \left\{\|A X-U\|_{2}+\alpha\|X\|_{1}\right\}$ & Lasso & Iterative & Yes & Non-Integer & No \\
This contribution & $\arg \min \left\|f^{T} X\right\|$ & ILP & Iterative & Yes & Integer & Yes \\
\hline
\end{tabular}

suboptimal solutions to (13) and (14) or attempt to get close to the optimality. The BB algorithm has proven to be a superior method among them in solving a seemingly intractable ILP problem. After extensively improving by the presolve and cutting plane techniques, branch-and-cut (BC) emerges [32]. Benefiting from a collection of problem reduction, a tighter convex problem relaxation, and parallel computing the solving speed has improved significantly. In this article, GUROBI optimization, which incorporates $\mathrm{BC}$, heuristic, and parallelism techniques are used to complete the solving process. In particular, the subroutine "intlinprog.m" in GUROBI has been used as the ILP solver.

In summary, phase ambiguity correction after SPU based on APSP network consists of four steps as shown in the workflow diagram (see Fig. 1): 1) point by point phase closure check: $(8)$; 2) constructing linear equation; 3) performing ILP for points with non-zero phase closure: (13) and (14); and 4) adding back the solved ambiguity.

\section{Results and Analysis: Synthetic Data}

\section{A. Performance of APSP Network in Spatial PU}

Simulated observations provide a great opportunity for testing the performance of APSP and comparison with other 2-D SPU methods, as the true values are available. Our synthetic observation consists of 10000 sparse points, randomly distributed in a rectangle of size $2000 \times 2000$ [see Fig. 2(a)]. 50 interferorgams are simulated by randomly generated temporal and perpendicular baselines. The linear deformation rate is simulated by the peak function of MATLAB, shown in Fig. 2(a). The simulated phase of the atmospheric and noise components of the 50th interferogram are shown in Fig. 2(b) and (c), respectively. In addition, the phase noise is simulated by $\gamma=0.3$ [33]. Fig. 2(d) shows the 50th simulated interferogram containing deformation, atmospheric, and noise elements. In this experiment, we compare the APSP-Based SPU with the original SPU based on the MCF [34].

First, we investigate the performance of the APSP algorithm in updating the Delaunay network. Comparing Fig. 2(i) with Fig. 2(e), the temporal coherence of the edges has improved significantly by using the APSP approach. Moreover, the unwrapped phase resulting from implementing these two approaches [see Fig. 2(f) and (j)] show notable differences. In particular, the unwrapping errors are significantly lower in the results obtained from the APSP method [see Fig. 2(k)] when compared to that from MCF, based on the Delaunay triangulation [see Fig. 2(g)]. Errors mainly occur where the deformation gradients are large. Therefore, the reduction of the unwrapping errors shows that the updated APSP network, which considers phase differences through introducing temporal coherence can more easily satisfy the phase continuity assumption. Lastly, we compare the sum of absolute values of the ambiguity integer cycles in 50 interferograms. One ambiguity integer cycle means an unwrapping error of $2 \pi$. Results of APSP in Fig. 2(1) indicate a higher accuracy than that of Delaunay in Fig. 2(h), especially in the high deformation gradient regions.

Using the simulated interferograms, we conducted a statistical test to evaluate how much PU errors are reduced through APSP. In this statistical test, the coherence is set to different values ranging from 0.05 to 0.95 . The simulation is repeated 1000 times and the percentages of points containing PU error are recorded in each simulation. The averaged results are shown in Fig. 3. It can be seen that, in most coherence cases, PU errors of APSP are about half of that from Delaunay, which further shows the effectiveness of APSP for reducing PU errors in the spatial domain.

\section{B. Performance of ILP in PU Ambiguity Correction}

To validate the phase ambiguity correction using ILP, a Monte-Carlo simulation test is carried out. The simulated SBAS graph is based on the sequential network [35]. In a sequential network, the nearest $N$ epochs are interconnected. In each phase simulation, the phase components consist of a linear trend of $50 \mathrm{~mm} / \mathrm{a}$, a seasonal signal with an amplitude of $20 \mathrm{~mm}$ and a noise component with a standard deviation of $10 \mathrm{~mm}$. In addition to these three components, $\pm 4 \pi$ phase ambiguities are randomly added to different percentages of the SBAS interferograms. This Monte-Carlo experiment is repeated 8000 times. Fig. 4(a) shows the statistical results.

In this test, the three PU methods are evaluated from two different aspects: 1) how many interferograms with PU errors are identified as PU error-free after correction ("Wrong to Right") and 2) how many PU error-free interferograms are misconstrued to contain PU errors after correction ("Right to Wrong"). It can be seen from Fig. 4(a), (c), and (e) that $L^{2}$ is the least effective correction method with the highest percentage of "Right to Wrong" across different percentages of interferograms with PU error introduced. The Lasso solver achieves a better result compared to the $L^{2}$ in terms of both percentage of corrected and the "Right to Wrong" interferograms. ILP solver, on the other hand, achieves the best results compared to both the $L^{2}$ and Lasso solvers, regardless of the percentage of interferograms with PU errors. After each correction, we also invert the SBAS phase to displacement 

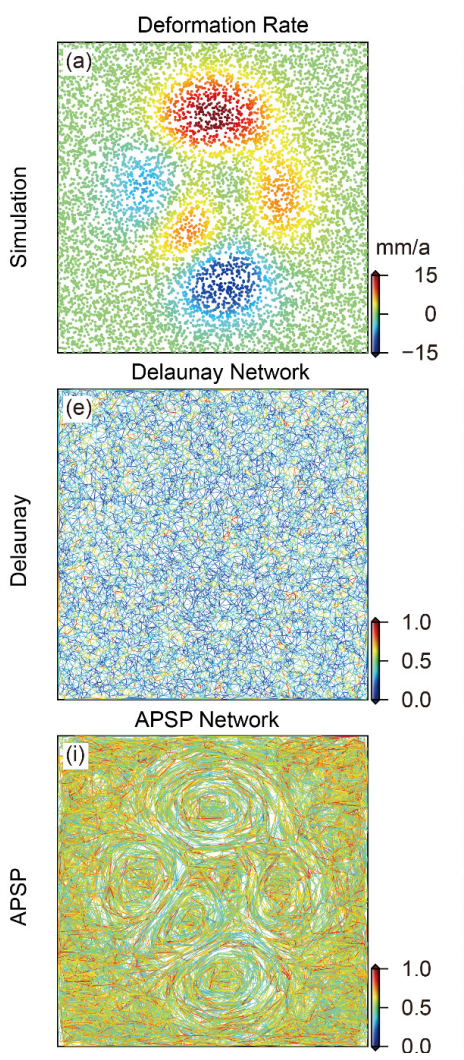
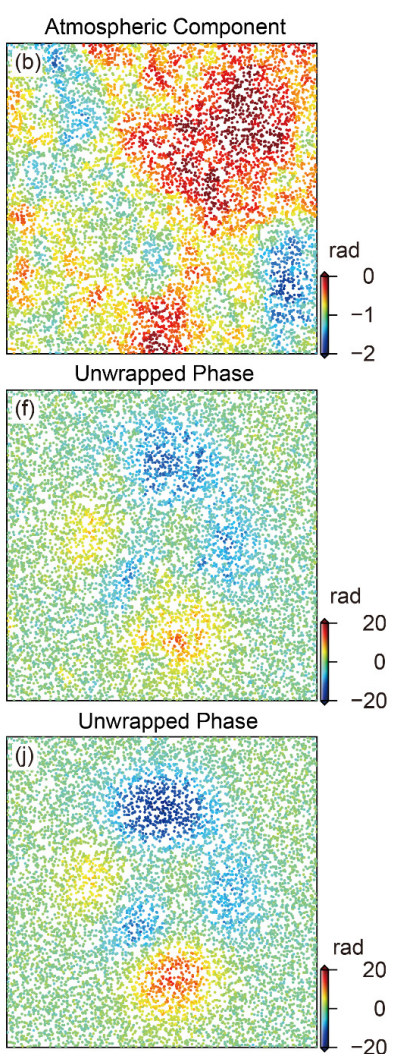
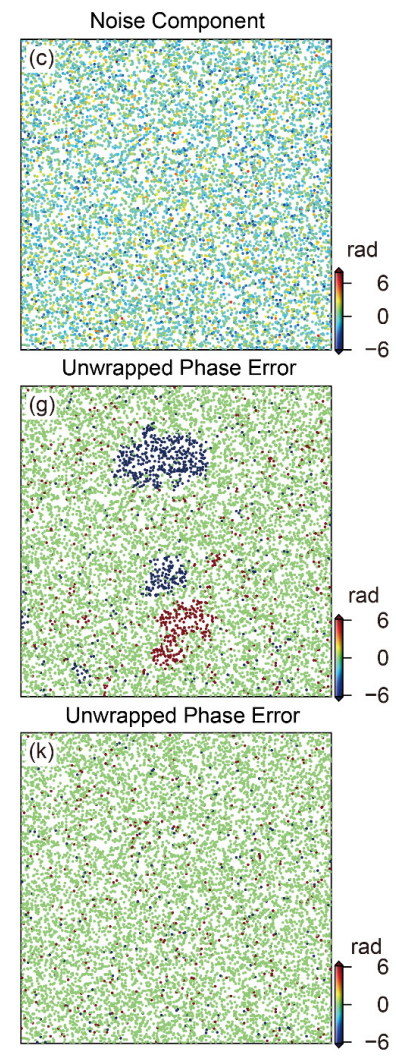

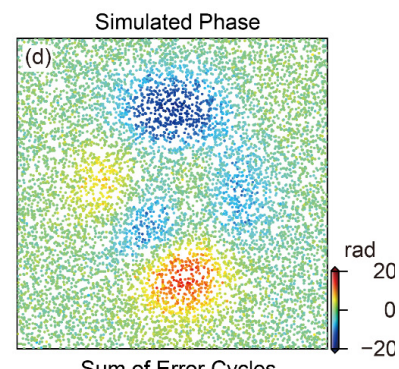

Sum of Error Cycles
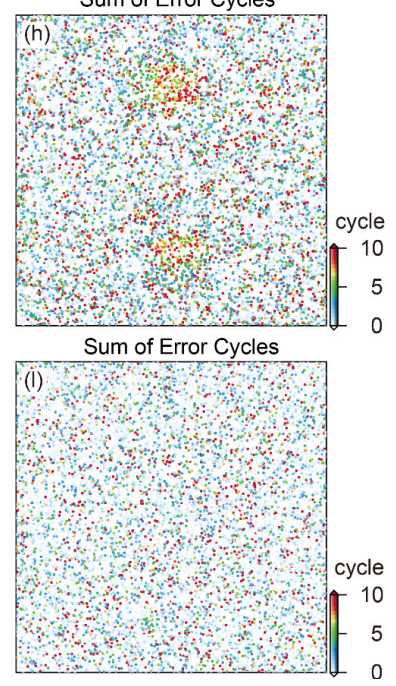

Fig. 2. (a) Simulated deformation rate. (b) Simulated atmospheric phase component of the 50th interferogram. (c) Simulated noise phase component of the 50th interferogram. (d) 50th simulated interferogram. (e)-(h) and (i)-(l) Results obtained from Delaunay network and APSP, respectively, (e) and (i) temporal coherence, (f) and (j) unwrapped phase, (g) and (k) unwrapped phase errors, and (h) and (l) sum of absolute ambiguity cycles in 50 interferograms.

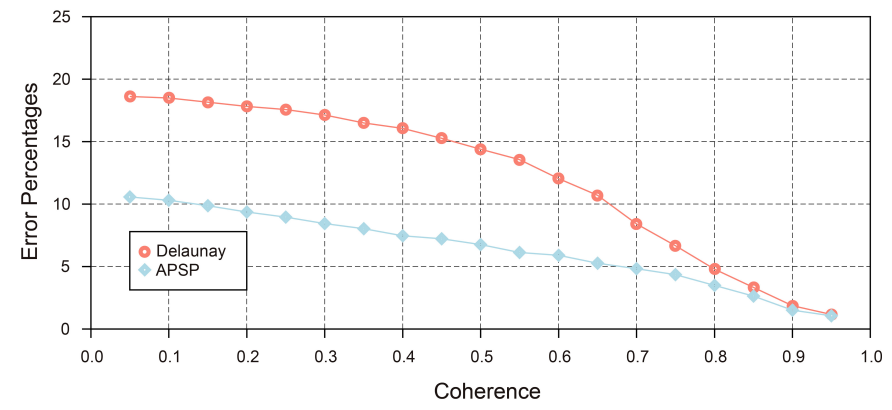

Fig. 3. Average ambiguity estimation error of 50 interferograms as a function of coherence. The salmon circles and light blue diamonds are associated with Delaunay network and APSP, respectively.

time series relative to a single reference and calculate the estimation error compared to the true values. Fig. 4(b), (d), and (f), respectively, shows the statistical results of the root mean square error (RMSE) of the estimated displacement series. It can be seen that the ILP outperforms the other two methods by correcting more unwrapping errors. From the test results, we find that ILP corrects the PU errors to a greater extent when the number of interferograms with PU errors is less than half the number of triangular loops in SBAS interferograms. This region is highlighted with a green rectangle in Fig. 4(a), (c), and (e) for this simulation. This is mainly due to the fact that the sparsity of $\left[X^{+} X^{-}\right]^{T}$ in this region is less than $\operatorname{Spark}([A-A]) / 2$, as mentioned in Section III-B. Although the accuracy of ILP decreases when the number of interferograms with PU errors exceeds this threshold, its performance is still superior to the other two methods. Also, the APSP network can provide SPU results with fewer PU errors. Therefore, the integration of APSP in SPU and ILP in temporal correction can make the final PU results more reliable.

\section{Results and Analysis: Real Data}

A stack of Sentinel-1 TOPS images over Lost Hills in southern California is chosen as the test data. The data set includes 57 SLCs from January 2017 to November 2018, acquired from a descending orbit. The temporal network we used in this experiment is the sequential network [35], in which the neighboring four acquisitions of each acquisition are interconnected. In PU with Delaunay and APSP network, the reciprocal of temporal coherence vector is treated as the edge cost.

\section{A. Performance of APSP Network}

To highlight the performance of APSP, Fig. 5 presents a comparison between unwrapped interferorgams based on the Delaunay network and the APSP network. It can be clearly seen that points in isolated regions of farmland (inside the dashed rectangle of Fig. 5) are clearly unwrapped incorrectly, while the results associated with the APSP network [see Fig. 5(b)] are almost error-free.

To further investigate the effectiveness of the APSP network, the number of non-closing triplets in unwrapped 


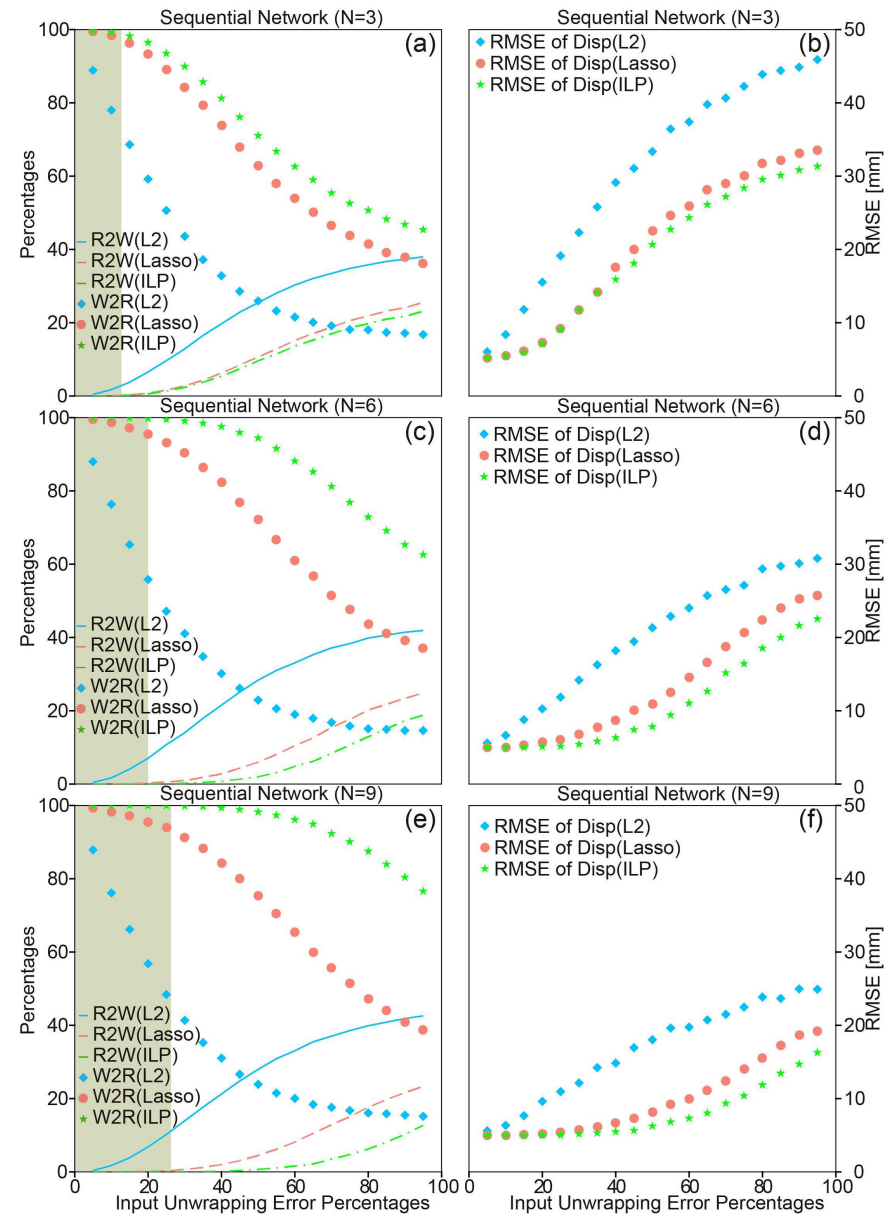

Fig. 4. Statistical results of three methods in phase ambiguity correction and displacement errors. (a), (c), and (e) Statistical results of PU error correction, respectively. (b), (d), and (f) Their respective RMSE of displacement inverted by corrected phases. "R2W" and "W2R" are abbreviations of "Wrong to Right" and "Right to Wrong," respectively. For (a), (c), and (e) $y$-axis means the percentages of "R2W" and "W2R." For (b), (d), and (f) $y$-axis means the RMSE of displacement time series for all epochs. For (a)-(f) $x$-axis denotes the percentages of the input unwrapping errors.

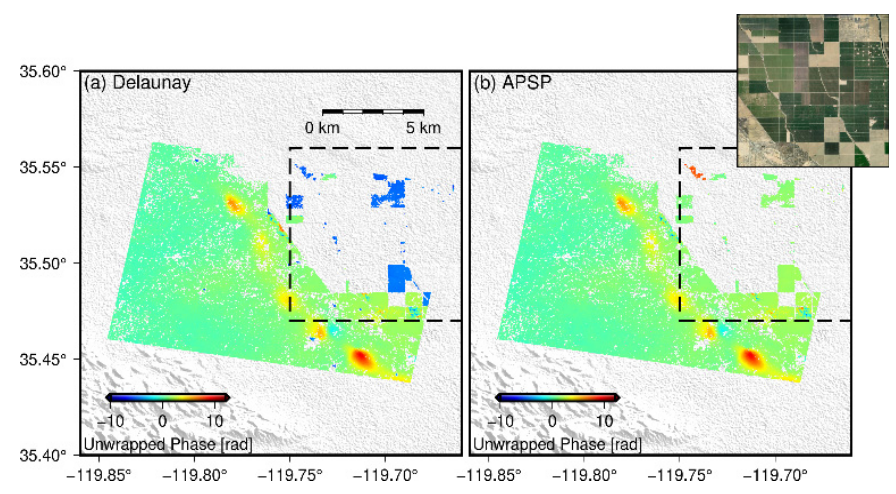

Fig. 5. Unwrapped Interferograms (20180806-20180830) based on (a) Delaunay and (b) APSP networks.

interferorgams is shown in Fig. 6. The smaller the proportion of the non-closing triplets to the total number of interferogram triplets, the higher the reliability of the unwrapped results. It can be seen in Fig. 6(a) that the non-closing loops in the results obtained from Delaunay triangulation far exceed that

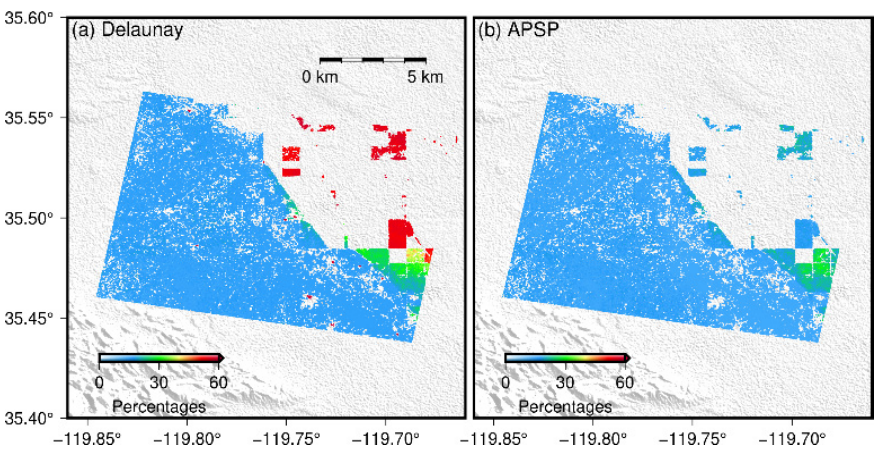

Fig. 6. Percentages of non-closing loops in all interferogram triplets. (a) Results from Delaunay triangulation. (b) Results from APSP network.
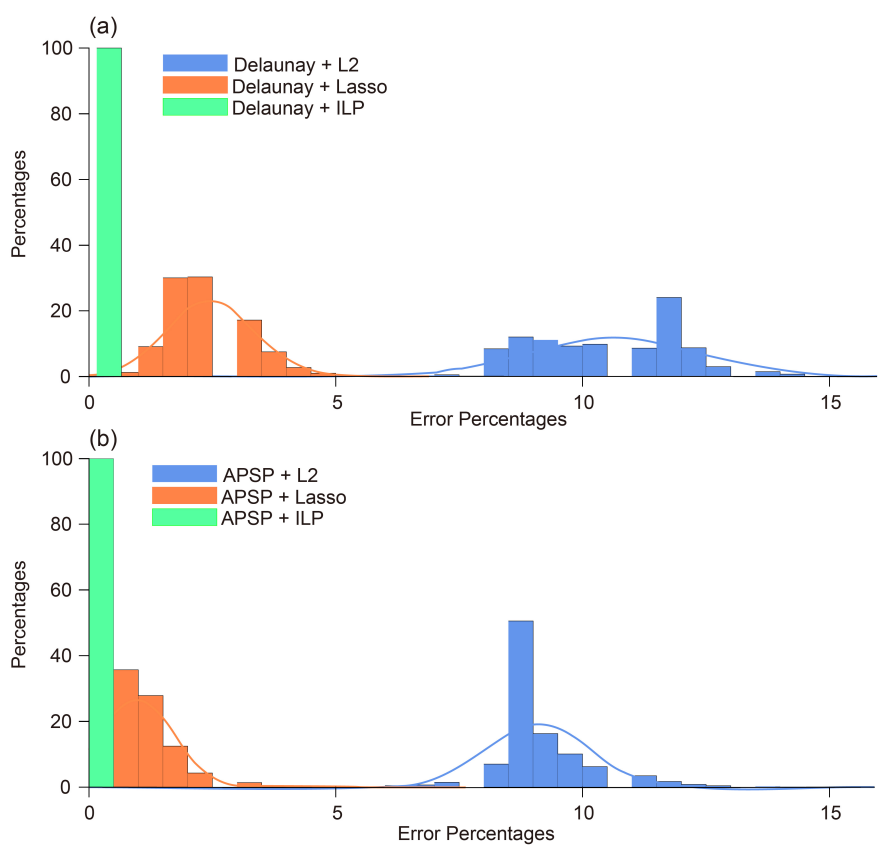

Fig. 7. Percentages of non-closing loops in all interferogram triplets after correction. (a) Results of Delaunay triangulation. (b) Results of APSP network.

of the APSP network, indicating the superior performance of the APSP network. Moreover, since only the networks are different in the PU process, we expect to see further improvement in the PU results by incorporating SPU. As shown in the simulation test (see Fig. 4), lower spatial unwrapping errors would be beneficial in the temporal correction step using every phase ambiguity correction method discussed in this study.

\section{B. Performance of ILP in Phase Ambiguity Correction}

To highlight the ILP's performance in correcting phase ambiguity, Fig. 7 presents a comparison of uncorrected proportions for all the interferogram triplets between different solvers. It can be seen that the percentage of non-closing triplets by using the Delaunay network is larger than that based on APSP, regardless of the PU error correction method. Using the Delaunay triangulation, error percentages of ILP are centered at $\sim 1 \%$, while L2 and Lasso are at $11 \%$ and $3 \%$, respectively, [see Fig. 7(a)]. However, using the ASPS network further reduces error percentages to near zero for ILP, 

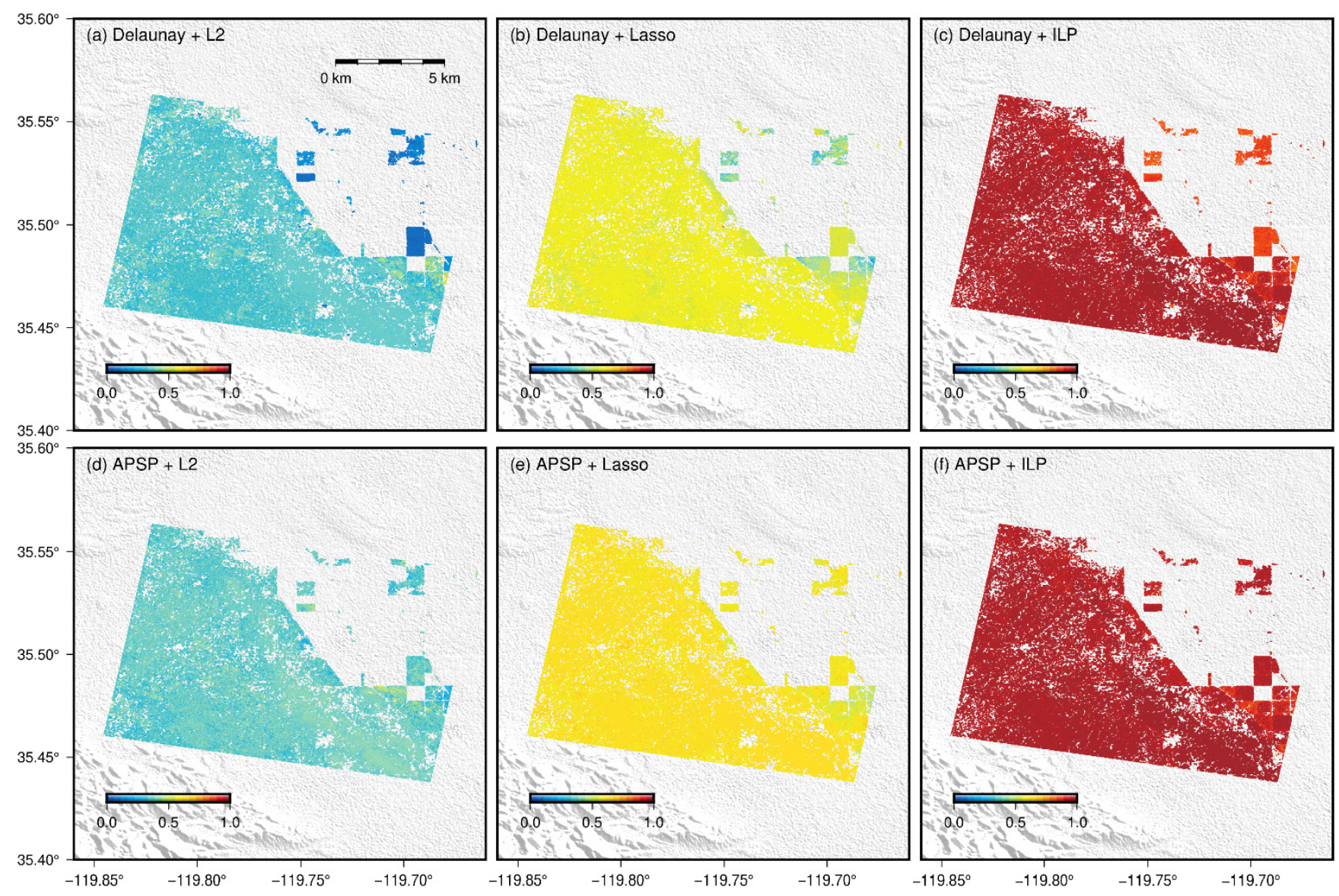

Fig. 8. Temporal coherence of all interferograms after correction using a combination of (a) Delaunay triangulation and L2 method, (b) Delaunay triangulation and Lasso method, (c) Delaunay triangulation and ILP method, (d) APSP network and L2 method, (e) APSP network and Lasso method, and (f) APSP network and ILP method.

and down to $2 \%$ and $8 \%$ for the results obtained from Lasso and L2, respectively, [see Fig. 7(b)].

To quantitatively demonstrate the effect of ambiguity correction methods, we calculate the temporal coherence $\gamma$ for each pixel, by replacing the wrapped phases with the unwrapped result in (1) as

$$
\gamma=\frac{1}{M}\left|\sum_{i=1}^{M} e^{j\left(\psi_{i}-\hat{\psi}_{i}\right)}\right|
$$

where $\hat{\psi}$ is the unwrapped phase of interferogram $i=$ $1, \ldots, M$.

Since the SBAS network of interferograms is fully connected, the remaining unwrapping errors can be considered as the principal source of noise compared to the magnitude of decorrelation noise. With this assumption, $\gamma=1$ indicates no remaining unwrapping errors in the interferogram set, while $\gamma<1$ implies the occurrence of uncorrected phase ambiguity.

Fig. 8(a)-(c), respectively, shows the temporal coherence of the three temporal correction methods after spatial ambiguity correction using Delaunay triangulation. Fig. 8(d)-(f) demonstrates the associated results by using the APSP network. As expected from Fig. 7, the coherence map associated with the results obtained from the APSP network is overall higher than that of Delaunay triangulation. This is mainly resulting from the effectiveness of the APSP network in reducing spatial PU errors. Moreover, the coherence maps by using ILP results in values close to 1 in both APSP and Delaunay. Whereas, the maximum coherence of $L^{2}$ and Lasso is close to 0.4 and 0.7. The superior performance of ILP results was expected from our simulation test (see Fig. 4).

For further validation of the performance of ILP, several corrected interferograms are presented in Fig. 9 for visual inspection. It can be seen that, regardless of the choice of the networking method used, the isolated regions with unwrapping errors are mostly corrected by using the ILP solver [see Fig. 9(c) and (f)]. In the results based on Delaunay triangulation [see Fig. 9(a) and (b)], L2 and Lasso failed to correct those unwrapping errors. For the results based on the APSP network [see Fig. 9(e) and (f)], although Lasso corrected most of the unwrapping errors, it still failed to correct the errors in some very small areas. These results further validate the performance of our proposed PU framework, based on the APSP network and ILP.

\section{Result Comparison With Stanford Method for Persistent Scatterers (StaMPS) 3-D PU Method}

Although in Sections V-A and V-B, we provided evidence for the superior performance of the proposed method compared to other techniques based on the Delaunay network and 

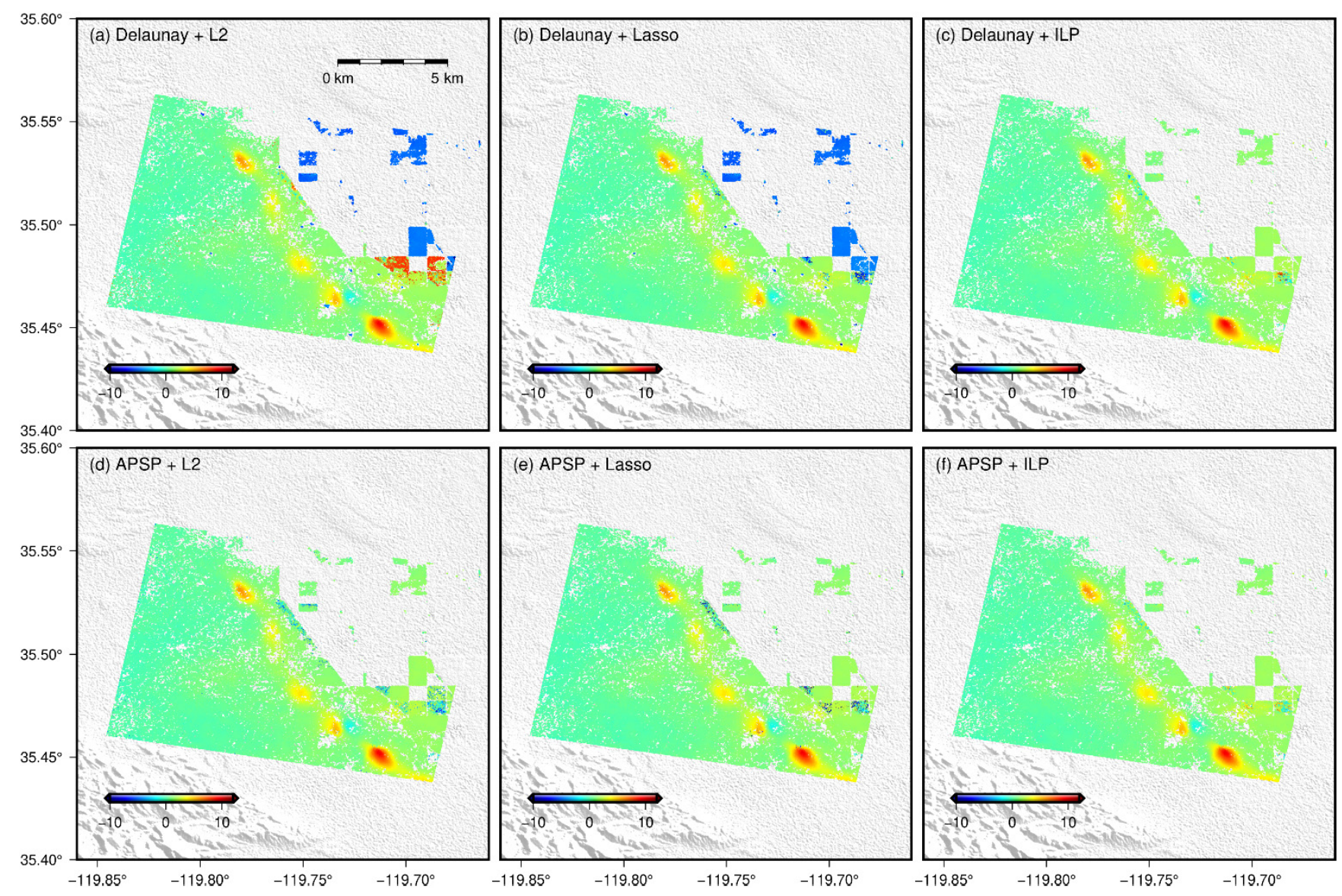

Fig. 9. Sample corrected interferograms (20180806-20180830) using methods based on a combination of (a) Delaunay triangulation and L2 method, (b) Delaunay triangulation and Lasso method, (c) Delaunay triangulation and ILP method, (d) APSP network and L2 method, (e) APSP network and Lasso method, and (f) APSP network and ILP method.

conventional PU error correction methods, further performance comparison with other 3-D methods is carried out here.

The 3-D PU subroutine [9] of the StaMPS [36] is widely accepted amongst the scientific community, and hence will be used to benchmark the performance of our 3-D PU method. Before PU, StaMPS 3-D method down-samples the wrapped phases to a user-defined grid resolution (in this study, we set the grid size to $50 \mathrm{~m}$ ). Next, it filters the gridded phase difference between neighboring pixels in the time domain to give an initial estimate of the unwrapped phase and the phase noise. Then a statistical-cost network-flow algorithm for PU (SNAPHU) method [2], [37] is used to search for the solution in space based on the previously filtered phase. Finally, ambiguity solutions will be interpolated at the location of the original pixels and added back to the wrapped interferograms.

We unwrapped 218 interferograms using the StaMPS 3-D method and calculated the associated temporal coherence using (15) and compared it to the result of our proposed method (APSP + ILP). Although the StaMPS 3-D method achieves good performance for most of the regions [see Fig. 10(a)], it is still outperformed by the proposed 3-D method, especially in the noisier area [see Fig. 10(b)]. This comparison further validates the effectiveness of our proposed 3-D PU framework.

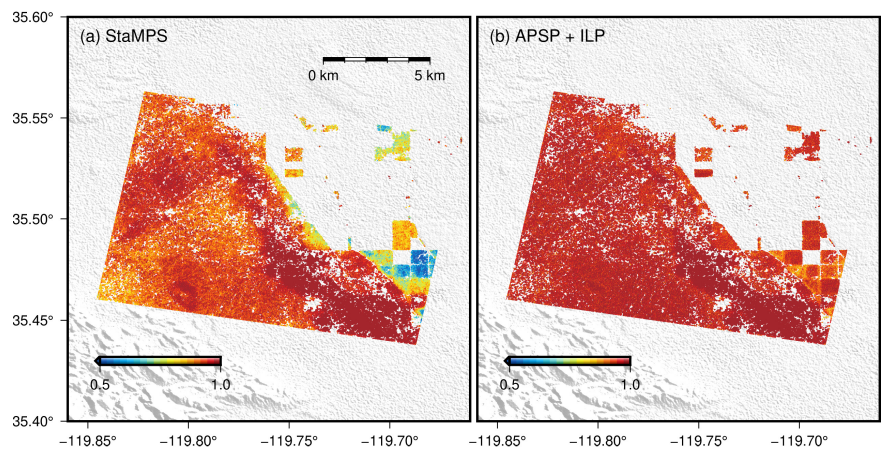

Fig. 10. Temporal coherence of unwrapped phase using (a) StaMPS and (b) proposed 3-D method (APSP + ILP).

\section{Processing Time}

For the APSP network, an additional time is required to search for the shortest paths between all the initial edges provided by the Delaunay triangulation. This is the most timeconsuming step in our method. With MATLAB 2018b software and an Intel i7 3.2-GHz processor, the generation of APSP network for 259361 sparse points takes 3452 s. However, in the following PU step in the spatial domain, the APSP network consisting of 781075 edges takes less time than the 


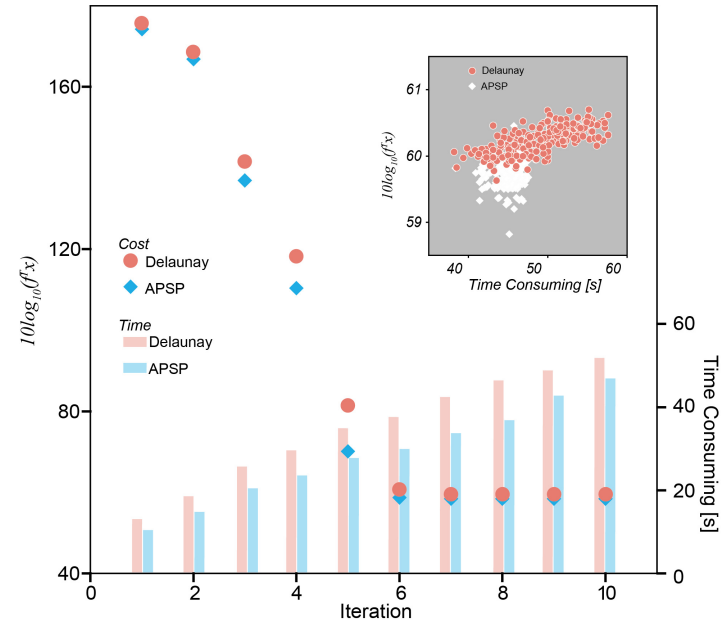

Fig. 11. Objective function cost and time consumption for ILP, and an example for the interferogram (20180806-20180830).

Delaunay network with 776399 edges. The APSP network operates $9892 \mathrm{~s}$ to solve ILP solutions for 218 interferograms, while the Delaunay network takes 10738 s. This is because there are more reliable edges in the APSP network, which are less likely to have phase ambiguities required to be compensated for. This, in effect, reduces the total objective function cost.

The subplot in Fig. 11 shows the APSP and Delaunay objective cost of 218 interferograms when using the ILP process. APSP outperforms Delaunay in objective cost and time consumption for most of the interferograms. Also, we present the iteration process and its relative computing time of the interferogram for 20180806-20180830 as an example. In iteration, the total cost and computing time of APSP are all less than that of Delaunay.

It is important to note that the part of time cost reduction comes from the edge constraint introduced in Section II. In the original SPU, phase cycles of all edges are required to be integrated using a flood fill algorithm, which increases the processing time significantly. This integration process is avoided in this article by modifications applied to the MCF objective function, enabling direct estimation of the phase ambiguity of all sparse points, which in turn improves the computational efficiency.

In temporal correction using different methods, $L^{2}$ and ILP solver have both shown great efficiency in terms of computation time. The $L^{2}$ took $1210 \mathrm{~s}$ to finish the phase ambiguity correction, while ILP took 1265 s. However, for finishing the phase ambiguity correction, Lasso consumes a considerably longer time, up to 81354 s. Overall, the combination of Delaunay and L2 consumed 11948 s. The combination of Delaunay and Lasso finished in 92092 s. The proposed 3-D method (APSP + ILP) took 14609 s, while the StaMPS 3-D method consumed only 4169 s, owing to the performed downsampling, which reduces the time required for SNAPHU. Although our method is slower than StaMPS, our proposed method can achieve high accuracy in a relatively efficient manner.

\section{CONCLUSION}

In this article, a 3-D (2-D + 1-D) PU method has been presented that integrates the APSP network on the spatial domain with ILP on the temporal domain. First, the APSP network has been proposed to replace the Delaunay network in the spatial domain. The APSP algorithm is used to maximize the temporal coherence of edges between two points. Thus, the phase difference induced by deformation and noise is reduced, making it easier to satisfy the phase continuity assumption. In addition, ILP based on the CS framework is first introduced in this article for phase ambiguity correction in the temporal domain. In addition to the great performance in terms of accuracy, another main advantage of this method is that it only modifies integer phase ambiguities without the change of the fractional part of the unwrapped phase. The effectiveness of the implemented integration of the APSP network and ILP has been validated using synthetic data and a real data set of Sentinel-1 TOPS interferograms over Lost Hills, CA region. In this article, we have two further findings.

1) APSP network can improve the accuracy and processing efficiency of PU. Compared to the Delaunay network, the accuracy improvement is $\sim 7 \%$ and the computational improvement is $\sim 4 \%$.

2) The proposed ILP PU error correction method can correct the unwrapping error to a greater extent if the number of interferograms with unwrapping errors is less than half the number of triangle loops in a SBAS graph. According to the conducted experiments, the time consumption of the proposed correction method is $\sim 2 \%$ of that of Lasso and is nearly equal to that of $L^{2}$. The accuracy improvement is $\sim 3 \%$ and $\sim 11 \%$ compared to $L^{2}$ and Lasso, respectively.

\section{APPENDIX}

This appendix aims to show how to construct incidence matrix $A$ and constraint matrix $D$ of a SBAS graph for phase ambiguity correction.

First, here are the definition of the notations used: acquisitions as vertex set $V$, interferograms as edges set $E_{N \times 1}(V)$, triangle loops set $\operatorname{Tri}_{M \times 1}(E)$, and the SBAS graph as the directed graph $\mathcal{G}($ Tri $)$.

Associated with a directed graph $\mathcal{G}$, we can define the incidence matrix $A_{M \times N}$ as

$$
\begin{aligned}
A_{M \times N}= & {\left[\begin{array}{ccccc}
a_{11} & a_{12} & a_{13} & \ldots & a_{1 N} \\
a_{21} & a_{22} & a_{23} & \ldots & a_{2 N} \\
\vdots & \vdots & \vdots & \vdots & \vdots \\
a_{M 1} & a_{M 2} & a_{M 3} & \ldots & a_{M N}
\end{array}\right] } \\
\text { s.t. } a_{i j}= & \left\{\begin{array}{ccc}
-1, & \text { if } E_{j} \text { is counter-clockwise direction in } \operatorname{Tr}_{i} \\
1, & \text { If } E_{j} \text { is clockwise direction in } \text { Tri }_{i} \\
0, & \text { otherwise }
\end{array}\right. \\
& (i=1,2,3, \ldots, M ; j=1,2,3, \ldots, N)
\end{aligned}
$$




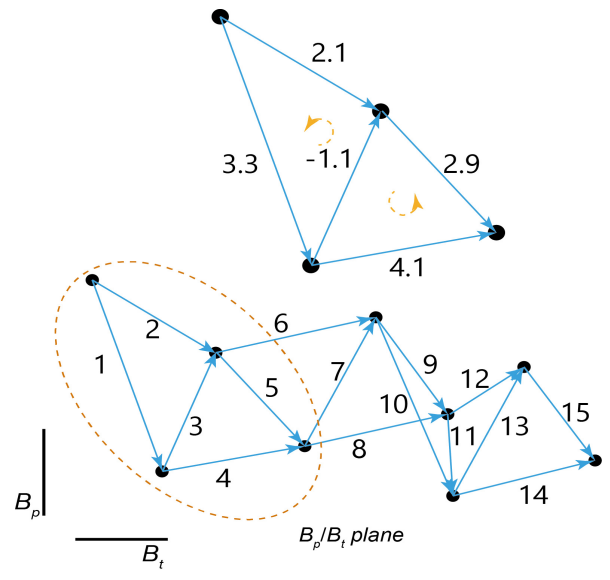

Fig. 12. Example of SBAS graph.

Example 1: The incidence matrix of the SBAS graph in Fig. 12 is depicted by

$$
A_{7 \times 15}=\left[\begin{array}{ccccccccccccccc}
1 & 1 & -1 & 0 & 0 & 0 & 0 & 0 & 0 & 0 & 0 & 0 & 0 & 0 & 0 \\
0 & 0 & -1 & 1 & -1 & 0 & 0 & 0 & 0 & 0 & 0 & 0 & 0 & 0 & 0 \\
0 & 0 & 0 & 0 & 1 & -1 & 1 & 0 & 0 & 0 & 0 & 0 & 0 & 0 & 0 \\
0 & 0 & 0 & 0 & 0 & 0 & -1 & 1 & -1 & 0 & 0 & 0 & 0 & 0 & 0 \\
0 & 0 & 0 & 0 & 0 & 0 & 0 & 0 & -1 & 1 & -1 & 0 & 0 & 0 & 0 \\
0 & 0 & 0 & 0 & 0 & 0 & 0 & 0 & 0 & 0 & 1 & -1 & 1 & 0 & 0 \\
0 & 0 & 0 & 0 & 0 & 0 & 0 & 0 & 0 & 0 & 0 & 0 & 1 & 1 & -1
\end{array}\right] .
$$

The constraint matrix $D$ denotes the correct unwrapped interferograms. As given in [10], the definition of a correct interferogram is the edge that appears in two triangle loops with no unwrapping errors. Besides, we need to add a constraint to ensure the solutions of these interferograms to be zero when several interferograms contain no unwrapping errors. Considering the example shown in Fig. 12, edge 3 can be described as an interferogram without unwrapping errors. Assuming edge 7, 11 also contain no unwrapping errors, the constraint matrix $D$ can be formulated as Example 2

$$
D_{3 \times 15}\left[\begin{array}{ccccccccccccccc}
0 & 0 & 1 & 0 & 0 & 0 & 0 & 0 & 0 & 0 & 0 & 0 & 0 & 0 & 0 \\
0 & 0 & 0 & 0 & 0 & 0 & 1 & 0 & 0 & 0 & 0 & 0 & 0 & 0 & 0 \\
0 & 0 & 0 & 0 & 0 & 0 & 0 & 0 & 0 & 0 & 1 & 0 & 0 & 0 & 0
\end{array}\right]
$$

\section{Appendix Obtaining the Code}

The APSP network-based spatial PU and CS-based PU error correction described here are a MATLAB-based application developed by a team of researchers, and we are happy to provide a copy to non-commercial researchers. The code is well documented and has been tested in different regions with different coherence conditions. However, it has not been through the same quality control procedure that is expected for commercial software. For using this software, some computing environments need to be configured (such as GUROBI software), for which guidance is provided. If you would like to obtain a copy or are interested in collaborative researches, contact us at the following e-mail: jspcmazhangfeng@hhu.edu.cn.

\section{ACKNOWLEDGMENT}

The Sentinel-1 data were provided by ESA/Copernicus. Thanks to GUROBI company, Beaverton, OR, USA, for providing the academic license of GUROBI software (License ID: 413287). All figures in this article were drawn by GMT6.1.0 software. The authors would like to thank three anonymous reviewers for their valuable comments.

\section{REFERENCES}

[1] H. Yu, Y. Lan, Z. Yuan, J. Xu, and H. Lee, "Phase unwrapping in InSAR: A review," IEEE Geosci. Remote Sens. Mag., vol. 7, no. 1, pp. 40-58, Mar. 2019.

[2] C. W. Chen and H. A. Zebker, "Two-dimensional phase unwrapping with use of statistical models for cost functions in nonlinear optimization," J. Opt. Soc. Amer. A, Opt. Image Sci., vol. 18, no. 2, pp. 338-351, 2001.

[3] R. Li, X. Lv, and Y. Yun, "A network-optimization-based $L^{1}$-norm sparse 2-D phase unwrapping method for persistent scatterer interferometry," IEEE Geosci. Remote Sens. Lett., vol. 15, no. 5, pp. 709-713, May 2018.

[4] D. C. Ghiglia and L. A. Romero, "Minimum Lp-norm two-dimensional phase unwrapping," J. Opt. Soc. Amer. A, Opt. Image Sci., vol. 13, no. 10, pp. 1999-2013, Oct. 1996.

[5] M. Costantini, "A novel phase unwrapping method based on network programming," IEEE Trans. Geosci. Remote Sens., vol. 36, no. 3, pp. 813-821, May 1998.

[6] T. J. Flynn, "Two-dimensional phase unwrapping with minimum weighted discontinuity," J. Opt. Soc. Amer. A, Opt. Image Sci., vol. 14, no. 10 , pp. $2692-2701,1997$.

[7] A. Pepe and R. Lanari, "On the extension of the minimum cost flow algorithm for phase unwrapping of multitemporal differential SAR interferograms," IEEE Trans. Geosci. Remote Sens., vol. 44, no. 9, pp. 2374-2383, Sep. 2006.

[8] M. Costantini, F. Malvarosa, and F. Minati, "A general formulation for redundant integration of finite differences and phase unwrapping on a sparse multidimensional domain," IEEE Trans. Geosci. Remote Sens., vol. 50, no. 3, pp. 758-768, Mar. 2012.

[9] A. Hooper and H. A. Zebker, "Phase unwrapping in three dimensions with application to InSAR time series," J. Opt. Soc. Amer. A, Opt. Image Sci., vol. 24, no. 9, pp. 2737-2747, Aug. 2007.

[10] J. Biggs, T. Wright, Z. Lu, and B. Parsons, "Multi-interferogram method for measuring interseismic deformation: Denali Fault, Alaska," Geophys. J. Int., vol. 170, no. 3, pp. 1165-1179, Sep. 2007.

[11] H. Fattahi, "Geodetic imaging of tectonic deformation with InSAR," Ph.D. dissertation, Dept. Marine Geol. Geophys., Univ. Miami, Ann Arbor, MI, USA, 2015, Art. no. 3719950.

[12] X. Xu and D. T. Sandwell, "Toward absolute phase change recovery with InSAR: Correcting for earth tides and phase unwrapping ambiguities," IEEE Trans. Geosci. Remote Sens., vol. 58, no. 1, pp. 726-733, Jan. 2020.

[13] T. Park and G. Casella, "The Bayesian lasso," J. Amer. Stat. Assoc., vol. 103, no. 482, pp. 681-686, 2008.

[14] Z. Yunjun, H. Fattahi, and F. Amelung, "Small baseline InSAR time series analysis: Unwrapping error correction and noise reduction," Comput. Geosci., vol. 133, Dec. 2019, Art. no. 104331.

[15] M. Jiang and A. M. Guarnieri, "Distributed scatterer interferometry with the refinement of spatiotemporal coherence," IEEE Trans. Geosci. Remote Sens., vol. 58, no. 6, pp. 3977-3987, Jun. 2020.

[16] R. W. Floyd, "Algorithm 97: Shortest path," Commun. ACM, vol. 5, no. 6 , p. 345 , Jun. 1962.

[17] M. Jiang, "Sentinel-1 TOPS co-registration over low-coherence areas and its application to velocity estimation using the all pairs shortest path algorithm," J. Geodesy, vol. 94, no. 10, p. 95, 2020.

[18] A. P. Shanker and H. Zebker, "Edgelist phase unwrapping algorithm for time series InSAR analysis," J. Opt. Soc. Amer. A, Opt. Image Sci., vol. 27, no. 3, pp. 605-612, Mar. 2010.

[19] J. E. Fowler, "Compressive-projection principal component analysis," IEEE Trans. Image Process., vol. 18, no. 10, pp. 2230-2242, Oct. 2009.

[20] E. J. Candes and M. B. Wakin, "An introduction to compressive sampling," IEEE Signal Process. Mag., vol. 25, no. 2, pp. 21-30, Mar. 2008.

[21] E. J. Candes and T. Tao, "Near-optimal signal recovery from random projections: Universal encoding strategies?" IEEE Trans. Inf. Theory, vol. 52, no. 12, pp. 5406-5425, Dec. 2006.

[22] D. L. Donoho, "Compressed sensing," IEEE Trans. Inf. Theory, vol. 52, no. 4, pp. 1289-1306, Apr. 2006. 
[23] E. J. Candes and T. Tao, "Decoding by linear programming," IEEE Trans. Inf. Theory, vol. 51, no. 12, pp. 4203-4215, Dec. 2005.

[24] M. Zhao, M. D. Kaba, R. Vidal, D. P. Robinson, and E. Mallada, "Sparse recovery over graph incidence matrices," in Proc. IEEE Conf. Decis. Control (CDC), Dec. 2018, pp. 364-371.

[25] P. S. R. Murty, "Chapter 2-Graph theory," in Power Systems Analysis, 2nd ed., P. S. R. Murty, Ed. Boston, MA, USA: Butterworth, 2017, pp. 7-17.

[26] P. S. R. Murty, "Chapter 3-Incidence matrices," in Power Systems Analysis, 2nd ed., P. S. R. Murty, Ed. Boston, MA, USA: Butterworth, 2017, pp. 19-33.

[27] M. Sipser, Introduction to the Theory of Computation. New York, NY, USA: Association for Computing Machinery, 1996, pp. 27-29.

[28] S. S. Chen, D. L. Donoho, and M. A. Saunders, "Atomic decomposition by basis pursuit," SIAM Rev., vol. 43, no. 1, pp. 129-159, Jan. 2001.

[29] D. L. Donoho, "For most large underdetermined systems of linear equations the minimal $\ell_{1}$-norm solution is also the sparsest solution," Commun. Pure Appl. Math., vol. 59, no. 6, pp. 797-829, 2006.

[30] J. Marshall and J. Bethel, "Basic concepts of $\mathrm{L}_{1}$ norm minimization for surveying applications," J. Surv. Eng., vol. 122, no. 4, pp. 168-179, 1996.

[31] S. P. Bradley, A. C. Hax, and T. L. Magnanti, Applied Mathematical Programming. Reading, MA, USA: Addison-Wesley, 1977.

[32] L. Ladányi, T. K. Ralphs, and L. E. Trotter, "Branch, cut, and price: Sequential and parallel," in Computational Combinatorial Optimization: Optimal or Provably Near-Optimal Solutions, M. Jünger and D. Naddef, Eds. Berlin, Germany: Springer, 2001, pp. 223-260.

[33] R. F. Hanssen, Radar Interferometry: Data Interpretation and Error Analysis. Dordrecht, The Netherlands: Springer, 2001.

[34] M. Costantini and P. A. Rosen, "A generalized phase unwrapping approach for sparse data," in Proc. IEEE Int. Geosci. Remote Sens. Symp. (IGARSS), vol. 1, Jun. 1999, pp. 267-269.

[35] H. Fattahi, P. Agram, and M. Simons, "A network-based enhanced spectral diversity approach for TOPS time-series analysis," IEEE Trans. Geosci. Remote Sens., vol. 55, no. 2, pp. 777-786, Feb. 2017.

[36] A. Hooper, "A multi-temporal InSAR method incorporating both persistent scatterer and small baseline approaches," Geophys. Res. Lett., vol. 35, no. 16, Aug. 2008, Art. no. L16302.

[37] C. W. Chen and H. A. Zebker, "Phase unwrapping for large SAR interferograms: Statistical segmentation and generalized network models," IEEE Trans. Geosci. Remote Sens., vol. 40, no. 8, pp. 1709-1719, Aug. 2002.

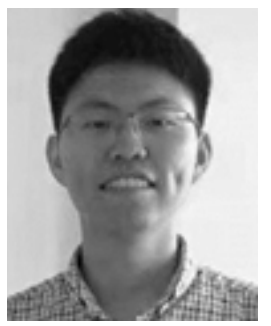

Zhang-Feng Ma (Student Member, IEEE) was born in Nantong, China, in 1995. He received the B.S. degree in geomatics engineering from the Nanjing University of Technology, Nanjing, China, in 2017, and the M.Sc. degree in geomatics engineering from Hohai University, Nanjing, in 2019, where he is pursuing the Ph.D. degree.

His research interests include interferometric synthetic aperture radar and its application for monitoring and modeling crustal deformation.

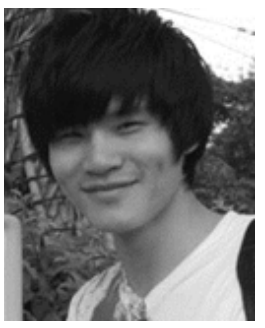

Mi Jiang (Senior Member, IEEE) was born in Nanjing, China, in 1982. He received the B.S. degree in computational mathematics from the Nanjing University of Technology, Nanjing, in 2005, the M.Sc. degree in radar remote sensing from Central South University, Changsha, China, in 2009, and the Ph.D. degree from The Hong Kong Polytechnic University, Hong Kong, in 2014.

$\mathrm{He}$ is an Associate Professor with the School of Geospatial Engineering and Science, Sun Yat-Sen University, Zhuhai, China. His research interest includes statistical inference with an emphasis on interferometric synthetic aperture radar processing

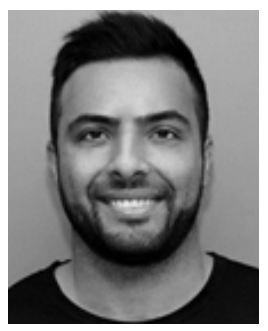

Mostafa Khoshmanesh received the B.S. degree in civil engineering from the University of Zanjan, Zanjan, Iran, in 2010, the M.Sc. degree in civil engineering from the $\mathrm{K}$. N. Toosi University of Technology, Tehran, Iran, in 2013, and the Ph.D. degree from Arizona State University, Tempe, AZ, USA, in 2018.

$\mathrm{He}$ is a Post-Doctoral Scholar with the California Institute of Technology, Pasadena, CA, USA. His research interests include satellite geodesy [SAR interferometry (InSAR) and global positioning system (GPS)] and modeling the processes associated with crustal deformation, such as earthquakes, induced seismicity, and landslides.

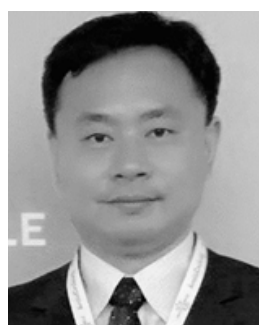

Xiao Cheng received the M.S. degree in geodesy and measurement engineering from Wuhan University, Wuhan, China, in 2001, and the Ph.D. degree in cartography and geographic information systems from the Chinese Academy of Sciences, Beijing, China, in 2004

$\mathrm{He}$ is a Professor and the Dean of the School of Geospatial Engineering and Science, Sun Yat-sen University, Guangzhou, China. He has authored more than 60 peer-reviewed articles. His research interest includes the observation of climate change impacts in polar regions, such as ice sheets, ice shelves, and sea ice using remote sensing technology.

Dr. Cheng is a Council Member of the Chinese National Committee of Polar Experts and the Secretary-General of the University Corporation for Polar Research (UCPR). 